Marjolein Vercruyssen

Catherine Cox

Ignace Naert

Reinhilde Jacobs

Wim Teughels*

Marc Quirynen

\title{
Accuracy and patient-centered outcome variables in guided implant surgery: a RCT comparing immediate with delayed loading
}

Key words: clinical research, clinical trials, CT Imaging, guided surgery, immediate loading, patient centered outcomes, prosthodontics

\begin{abstract}
Marjolein Vercruyssen, Catherine Cox, Wim
Teughels, Marc Quirynen, Department of Oral

Health Sciences, Periodontology, Faculty of

Medicine, Catholic University Leuven, University

Hospitals Leuven, Leuven, Belgium

Ignace Naert, Department of Oral Health Sciences,

Prosthetic Dentistry, Faculty of Medicine, Catholic

University Leuven, University Hospitals Leuven,

Leuven, Belgium

Reinhilde Jacobs, OMFS IMPATH research group,

Department of Imaging \& Pathology, Faculty of

Medicine, University of Leuven and Oral \&

Maxillofacial Surgery, University Hospitals Leuven,

Leuven, Belgium
\end{abstract}

Corresponding author:

Marjolein Vercruyssen

Department of Periodontology

Catholic University Leuven

Kapucijnenvoer 7

Leuven, Belgium

Tel.: +32 136332483

e-mail: marjo_vercruyssen@yahoo.com

Abstract

Aim: To assess the accuracy and patient-centered outcome of a novel guided surgery system for placing implants in an edentulous maxilla.

Material and methods: Fifteen consecutive patients with sufficient bone to place six implants in the maxilla were randomly assigned to the immediate loading (with delivery of the final prosthesis within $24 \mathrm{~h}$ ) or the delayed loading treatment group. Accuracy was assessed by matching the planning CT with a postoperative CBCT. Patient-centered outcome measures were the Dutch version of the McGill Pain Questionnaire (MPQ-DLV), the health-related quality of life instrument (HRQOL), visual analog scales (VAS), the duration of the procedure, and the analgesic doses taken each day.

Results: A mean deviation was found at the entry point of $0.9 \mathrm{~mm}$ (range: $0.1-4.5$, median 0.8 ) and of $1.2 \mathrm{~mm}$ (range: $0.2-4.9$, median 1.1) at the apex, and an angular deviation of $2.7^{\circ}$ (range: $0.0-6.6^{\circ}$, median 2.3 ) was observed. The mean vertical deviation was $0.5 \mathrm{~mm}$ (range: $0.0-3.2$, median 0.4 ), and in a horizontal direction, this was $0.7 \mathrm{~mm}$ (range: $0.1-3.1$, median 0.6 ). The mean deviation in mesio-distal direction was $0.5 \mathrm{~mm}$ (range: $0.0-2.3$, median 0.4 ) and in bucco-lingual direction $0.5 \mathrm{~mm} \pm 0.4$ (range: $0.0-2.2$, median 0.3 ). No statistical differences could be shown between treatment groups on pain response (MPQ-DLV), treatment perception (VAS), number or kind of pain killers, or for the HRQOLI instrument.

Conclusion: The accuracy of a novel CT-based guide is comparable to the accuracy data of other systems. Within the limitations of this study, no difference could be found in patient-centered outcome variables after immediate or delayed loading.

For the treatment of edentulous patients with guided surgery, significant variations can be observed (Vercruyssen et al. 2014d). In case of a flapless approach, the exposure of the bone during the drilling procedure is kept to a minimum. This is achieved by applying a punch-technique or a small crestal incision. In case of a bone-supported guide, the guide is positioned on the jawbone after reflecting of a mucoperiosteal flap with a crestal incision. In addition, for drill guidance, various handling procedures also exist. Some use different templates with sleeves with increasing diameter for an individual surgical case, while others use removable sleeve inserts in one single template (Van Assche et al. 2012).
Furthermore, different types of sleeve inserts are available. Handhold sleeve inserts are designed as a spoon and are stabilized by the surgeon's hand. Drill-hold sleeve inserts or sleeve on drills are only attached to the drill (Koop et al. 2012). Some systems designed special drills or drill stops to allow depth control, while others have indication lines on the drills. After the preparation of the implant osteotomy, some systems allow a guided insertion of the implant (fully guided implant placement), while for other systems, the template has to be removed and the implant is inserted freehanded.

The accuracy of the entire guided procedure is defined as the deviation between the 
position of the placed implant and the planned implant and is a summation of all individual errors (Vercruyssen et al. 2008). In this study, a novel guided surgery system (ExpertEase ${ }^{\mathrm{TM}}$ Materialise Dental) is investigated. This system uses sleeves on drills. One possible source for error is the amount of deviation during drilling due to the tolerance of the drill in the sleeve insert. In an in vitro study (Koop et al. 2012), we tested the tolerance within the sleeve inserts of different surgical guiding systems. In the latter study, the sleeve on drills gave for all measurements larger deviations than handhold sleeve inserts. However, in this study, a Plexiglas box was representing the bone and the drills were forced to the maximum in the left and the right direction. This is the first "clinical" study to determine the accuracy of this novel guided system.

Guided implant surgery is considered to be a treatment with maximum patient comfort and minimal patient morbidity (Lindeboom \& van Wijk 2010; Vercruyssen et al. 2014e). In a recent randomized clinical trial (Vercruyssen et al. 2014c), however, little difference could be found in the patient outcome variables between bone versus mucosa-supported or guided versus non-guided surgery. In the latter study, all patients were treated with a delayed loading protocol. In most clinical studies reporting on the outcome of guided surgery, an immediate loading protocol is applied (Hultin et al. 2012). Besides the accuracy assessment, the present randomized clinical trial also aimed to compare the patient-centered outcome variables of immediate and delayed loading.

\section{Material and methods}

\section{Patients}

Fifteen patients with sufficient bone volume to place six implants in the edentulous upper jaw (mean age $=60$ year, 12 males, 3 females, 2 smokers) were consecutively recruited and randomly assigned to the immediate loading (with delivery of the final prosthesis within $24 \mathrm{~h}$ ) or the delayed loading treatment group. For inclusion in the study, subjects had to fulfill all of the inclusion and exclusion criteria (Table 1). The study was approved by the ethical committee of the KU Leuven University Hospital (B32220096198).

\section{Planning procedure}

A scan prosthesis was prepared at the prosthetic department of the University Hospital KU Leuven containing all information for the prosthetic restoration. If the existing denture fulfilled these conditions, this denture was

\section{Table 1. The inclusion and exclusion criteria}

\begin{tabular}{cl}
\hline Inclusion criteria \\
1 & Provision of informed consent \\
2 & 18 years or older \\
3 & Extraction sockets should have healed at least 4 months \\
4 & Sufficient bone volume to place six implants in the maxilla \\
5 & No previous bone augmentation procedures \\
6 & The mandible can be any kind of dentition as long as a well-distributed contact relationship with \\
7 & the new prosthesis in the maxilla can be established. \\
Exclusion criteria & Physical or psychological disorders prohibiting implant treatment \\
2 & Heavy smoking (>10 cigarettes/day) \\
3 & Present alcohol and/or drug abuse \\
4 & Physical handicap that may interfere with the ability to perform oral hygiene \\
\hline
\end{tabular}

transformed into a scan prosthesis. To secure an optimal fit of the scan prosthesis during the scanning process, a bite index in centric relation was prepared in putty material (SheraExact ${ }^{\circledR} 85$, Shera $\mathrm{GmbH} \&$ Co., Lemförde, Germany). A MSCT scan (Somatom Definition Flash $^{\circledR}$, Siemens, Erlangen Germany, at $120 \mathrm{kV}$ and $90 \mathrm{mAs}, 0.6 \mathrm{~mm}$ slice thickness, voxel size $330 \mu \mathrm{m}$ ) was made of the patient with the scan prosthesis and bite index positioned in the mouth. A MSCT was used because the initial protocol demanded the measurement of Hounsfield Units (which is not possible with CBCT), and therefore, a dose-reduced protocol was applied (Jacobs \& Quirynen 2014). A second scanning was performed of the prosthesis alone, with altered exposure parameters to also visualize the denture (Verstreken et al. 1996, 1998). Both sets of DICOM images were imported using Simplant ${ }^{\circledR}$ software (Materialise Dental, Leuven, Belgium). The implants were planned in the most optimal position toward both the jawbone and the prosthetic demands.

Patients were only enrolled when the planning indicated sufficient bone volume for successful implant placement without the need of a bone graft. At that moment, the patient was randomly assigned to one of the intervention groups. For all patients, the planning was transferred to the manufacture (Materialise Dental) for fabrication of a stereolithographic drill guide.

\section{Surgical protocol}

Surgery was performed under local anesthesia at the department of periodontology of the University Hospital KU Leuven. The stereolithographic guides were positioned on the mucosa using a bite index to secure a proper position. All the stereolithographic guides were fixed to the underlying bone by three to four anchor pins, equally distributed in the jaw. The drilling procedure involved the use of sleeves on drills (ExpertEase ${ }^{\mathrm{TM}}$, Materialise Dental) which are inserted in the surgical guide and guide the consecutive drills /with different diameters) in the correct position and angulation (Fig. 1). The drills had a physical stop, and the implant insertion was guided by a fixture mount that closely fitted the sleeve.

Ninety Ankylos implants ${ }^{\mathrm{TM}}$ (DENTSPLY Implants, Mölndal, Sweden) with diameter 3.5 or $4.5 \mathrm{~mm}$, and lengths ranging from 9.5 to $14 \mathrm{~mm}$ were inserted. All patients received analgesics (paracetamol $500 \mathrm{mg}$, three times per day), antibiotics (amoxicillin $500 \mathrm{mg}$, three times per day) for 5 days, and $0.12 \%$ chlorhexidine twice a day for 1 week. The duration of the procedure (in minutes) was registered. Ten days after the implant procedure, all patients returned for a clinical evaluation.

\section{Questionnaires}

To assess postoperative pain, the Dutch version of the McGill Pain Questionnaire



Fig. 1. Sleeve on drill. The drill is placed with the sleeve in the guide, than the drill moves through the sleeve. 
(MPQ-DLV) was used (Melzack 1975, 2005). This questionnaire was handed out as a diary, and patients were asked to fill in the questions every day, from day 1 until day 7. The MPQ-DLV consists of two parts. The first part of the questionnaire groups various pain descriptions according to their pain quality and ranks the descriptions of a certain quality according to their intensity. This gives two indices. The sum of the "number of words chosen" gives the NWC-T (range 0-20). Furthermore, the "pain rating index" (PRI-T) was calculated (range 0-63), this is the sum of the intensity ranking of the chosen pain words. The second part consists of $100 \mathrm{~mm}$ VAS to evaluate the amount of pain, ranging from 0 (no pain whatsoever) to 100 (worst pain imaginable) and the amount of swelling. The patients were asked to fill in the VAS at the day of surgery every $4 \mathrm{~h}$ and afterward daily. Patients were asked to score their pain three times; the pain they felt at the moment of questioning, and the minimum and maximum amount of pain they felt during the past 4 or $24 \mathrm{~h}$.

To assess the impact of the treatment on the quality of life, the health-related quality of life (HRQOL) instrument was used (Shugars et al. 1996). The HRQOL consists of 15 questions concerning the quality of life. The frequency of each symptom is scored on a six-point scale, and the scores are summed to yield a total HRQOL Index (HRQOLI) score (range 0-75), with higher scores on the HRQOLI being indicative of more postoperative discomfort and inconvenience in daily life. These questions were also part of the diary and to be filled in daily. The patients were also asked to document the number and the sort of analgesics taken each day. Furthermore, patients were asked to fill in VAS at the time of surgery and at the follow-up visit after 10 days, on the following questions: mean amount of pain during surgery, during the past $24 \mathrm{~h}$, whether they would repeat the procedure in the future, whether they found the duration of the procedure tolerable, and whether they would recommend the procedure to friends or family, ranging from 0 (maximal agreement) to 100 (maximal disagreement) (Nkenke et al. 2007).

\section{Prosthetic protocol}

For patients with the immediate loading protocol, the final prosthesis was prepared preoperatively at the department of prosthetic dentistry KU Leuven. At the dental laboratory, implant replica's were fixed in a duplicate of the surgical drill guide using the implant mounts in the drill sleeves and a working cast was poured. From this cast, a soft-tissue cast was prepared and mounted in an articulator to allow the premanufacturing of the final CrCo-reinforced hybrid removable prosthesis. The silicone key index was used to set the tooth arrangement according to the scan prosthesis. Directly after implant surgery, a final impression at implant level was performed. This allowed to fix the correct interimplant positioning and to correct for deviations between planning and operation. At the day of implant surgery, the patient left the department with healing abutments installed, without wearing his/her existing denture. In the laboratory, a second replica cast was poured and the final SynCone abutment selection was performed. The day after the implant surgery, the patient received the final prosthesis (a hybrid detachable prosthesis) (Fig. 2).

For the patients treated with the delayed loading protocol, cover screws were placed and patients were instructed not to wear their dentures during the first week after surgery. After 3 months of healing, abutments were installed and the final prosthetic superstructure was prepared.

\section{Accuracy of the technique}

Immediately after implant placement, a CBCT scan (Scanora ${ }^{\circledR}$ 3D, Soredex, Tuusula, Finland) was taken (at $85 \mathrm{kV}$ and $6 \mathrm{~mA}$, voxel size $250 \mu \mathrm{m}$ ) to check the final position of the implants. The postoperative data were matched to the preoperative planning data using the Mimics ${ }^{\circledR}$ software (Materialise Dental) to determine deviations in the three dimensions. This process is based on surface registration, which consists of a minimization of distances between both models (preop and postop) (Maes et al. 1997). In this case, an iterative closest point (ICP) algorithm was used to match the jaws. The global deviation is defined as the $3 \mathrm{D}$ distance between the

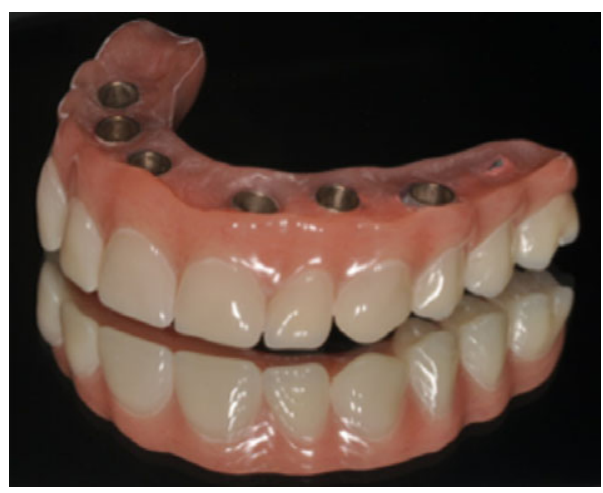

Fig. 2. A hybrid detachable prosthesis. coronal centers of the planned and placed implants. Moreover, a reference plane was set in bucco-lingual direction by which both the mesio-distal and bucco-lingual deviation could be calculated (Vercruyssen et al. 2014a) (Fig. 3).

The enrollment, assignment of the patients, the implant planning and the surgery were all performed by the same investigator (MV). The assessment of the accuracy was performed by another investigator (CC). The intra- and interexaminer variability of the procedure was determined in a previous study (Vercruyssen et al. 2014b).

\section{Statistical analysis}

The primary outcome variable was the deviation between the position of the planned and placed implant. The secondary outcome variables were the NWC-T index, the PRI-T index, the HRQOL instrument, the duration of the surgery, the VAS, and the amount of analgesic doses taken. The differences in patient-centered outcome variables between techniques were analyzed with a linear mixed model taking treatment as a fixed factor and patient as a random factor. Residual dot plots and normal quantile plots were used to assess the assumptions of the model. Contrasts were built to test the specific hypotheses, and a correction for simultaneous hypothesis testing was made according to Sidak (Šidák 1967). The level of significance was set at $\alpha=0.05$. For the secondary outcome variables from this study, a post hoc power analysis using the $\mathrm{N}$-factor was performed. The $\mathrm{N}$-factor is the percentage of data extra points needed to reach a level of significance ( $\alpha=0.05$ ) for the currently found difference /considering that when expanding the data set, the variability of data would remain the same). For the allocation, a computerized random number generator was used.

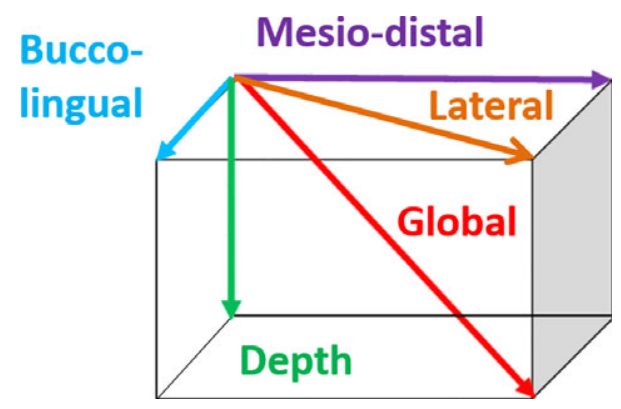

Fig. 3. Three dimensions of direction. Red: global coronal deviation, orange: lateral deviation, green: depth deviation, blue: bucco-lingual deviation, purple: mesiodistal deviation. 


\section{Results}

All the patients received their implants between February 2010 and December 2013. In the immediate loading, group 7 patients were enrolled versus 8 patients in the delayed loading group. Patient and implant demographics are shown in Table 2 . This study was ended preliminary, because insufficient number of patients could be found meeting all the inclusion criteria (sufficient bone volume). All implants were analyzed for the accuracy measurements, one implant from the delayed treatment group was lost before prosthesis installment due to non-integration.

A mean deviation was found at the entry point of $0.9 \mathrm{~mm}$ (range: $0.1-4.5$, median 0.8 ) and of $1.2 \mathrm{~mm}$ (range: $0.2-4.9$, median 1.1 ) at the apex, and an angular deviation of $2.7^{\circ}$ (range: 0.0-6.6 ${ }^{\circ}$, median 2.3) was observed. The mean vertical deviation was $0.5 \mathrm{~mm}$ (range: 0.0-3.2, median 0.4), and in a horizontal direction, this was $0.7 \mathrm{~mm}$ (range: $0.1-3.1$, median 0.6). The mean deviation in mesio-distal direction was $0.5 \mathrm{~mm}$ (range: 0.0-2.3, median 0.4) and in bucco-lingual direction $0.5 \mathrm{~mm} \pm 0.4$ (range: 0.0-2.2, median 0.3). In Table 3, the

Table 2. Patient and implant features

\begin{tabular}{|c|c|c|}
\hline Treatment group & Immediate & Delayed \\
\hline \multicolumn{3}{|c|}{ Parameter patient level $(n)$} \\
\hline $\begin{array}{l}\text { Total number of } \\
\text { patients }\end{array}$ & 7 & 8 \\
\hline $\begin{array}{l}\text { Gender (Male/ } \\
\text { Female) }\end{array}$ & $5 / 2$ & $7 / 1$ \\
\hline Age (Range) & $45-71$ & $49-70$ \\
\hline Smokers & 1 & 1 \\
\hline \multicolumn{3}{|c|}{ Parameter implant level ( $n)$} \\
\hline $\begin{array}{l}\text { Total number of } \\
\text { implants placed }\end{array}$ & 42 & 48 \\
\hline $\begin{array}{l}\text { Total number of } \\
\text { implants } \\
\text { analyzed }\end{array}$ & 42 & 48 \\
\hline $\begin{array}{l}\text { Total number of } \\
\text { implants lost } \\
\text { before loading }\end{array}$ & 0 & 1 \\
\hline $\begin{array}{l}\text { Bone quality } \\
\text { score } \\
(1 / 2 / 3 / 4)\end{array}$ & $0 / 18 / 24 / 0$ & $0 / 16 / 30 / 2$ \\
\hline
\end{tabular}

Table 3. Descriptive statistics (median, maximum and minimum positive and negative values of the depth, bucco-lingual, and mesio-dista deviation at the entry point of the implant $(\mathrm{mm})$

\begin{tabular}{lccc}
\hline & Depth $(\mathrm{mm})$ & MD $(\mathrm{mm})$ & LB $(\mathrm{mm})$ \\
\hline Min. & -3.18 & -2.25 & -2.17 \\
Max. & 1.79 & 1.33 & 1.65 \\
Median & 0.11 & -0.12 & -0.2 \\
\hline
\end{tabular}

Depth: placed deeper than planned/+ placed more occlusal than planned. Bucco-lingual (BL): placed more lingual than planned/+ placed more buccal than planned. Mesio-distal (MD): placed more to the right than planned/+ placed more to the left than planned.

Min., Minimum; Max., Maximum. minimum and maximum values of the vertical and horizontal deviations are presented.

The descriptive statistics for the secondary outcome variables are presented in Tables 4-6. The mean duration of the procedure was 82 min (range: 60-140). Over time, a significant reduction of the NWC-T and PRI-T index could be found for the immediate loading group after day $7(P \leq 0.01)$, and no difference was found for the delayed group.

Table 4. Descriptive statistics of the NWC-T, PRI-T index

\begin{tabular}{|c|c|c|}
\hline Treatment group & Delayed & Immediate \\
\hline \multicolumn{3}{|c|}{$\begin{array}{l}\text { Pain description list (MPQ-DLV) } \\
\text { NWC-T index }\end{array}$} \\
\hline \multicolumn{3}{|l|}{ NWC-T index } \\
\hline Day 1 & $0 ; 2$ (SD 2,5) & $0 ; 3$ (SD 1,9) \\
\hline Day 2 & $0 ; 3$ (SD 2,5) & $0 ; 1$ (SD 2,2) \\
\hline Day 3 & $0 ; 2(S D 2,4)$ & $0 ; 1$ (SD 1,5) \\
\hline Day 4 & $0 ; 2$ (SD 1,7) & $0 ; 0$ (SD 1,9) \\
\hline Day 5 & $0 ; 1$ (SD 1,6) & $0 ; 0$ (SD 1,9) \\
\hline Day 6 & $0 ; 1(\mathrm{SD} \mathrm{2,1)}$ & $0 ; 0$ (SD 1,9) \\
\hline Day 7 & $0 ; 0$ (SD 1,8) & $0 ; 0$ (SD 1,1) \\
\hline \multicolumn{3}{|l|}{ PRI-T index } \\
\hline Day 1 & $0 ; 3(\mathrm{SD} 2,7)$ & $1 ; 3$ (SD 2,9) \\
\hline Day 2 & $0 ; 4(\mathrm{SD} 2,9)$ & $1 ; 2$ (SD 2,6) \\
\hline Day 3 & $0 ; 3(\mathrm{SD} \mathrm{3,8)}$ & $0 ; 2$ (SD 1,7) \\
\hline Day 4 & $0 ; 3($ SD 2,7) & $0 ; 0$ (SD 3) \\
\hline Day 5 & $0 ; 1($ SD 1,7) & $0 ; 0$ (SD 2,6) \\
\hline Day 6 & $0 ; 1(\mathrm{SD} 3,1)$ & $0 ; 0$ (SD 2,3) \\
\hline Day 7 & $0 ; 0$ (SD 2,6) & $0 ; 0$ (SD 2,3) \\
\hline
\end{tabular}

The NWC-T index ranges from 0 to 20 and is indicative of the pain intensity based on the "number of words chosen" to prescribe the pain. The PRI-T index ranges from 0 to 63 and is the sum of the intensity ranking of the chosen pain words. The mean, median, and the standard deviation are presented.
For the HRQOL instrument (Table 5), over time, a significant reduction could be found at day 4 for the immediate loading group $(P=0.009)$, while for the delayed group, no difference was found. The evolution of the VAS scores over time is shown in Fig. 4. For the mean amount of pain at the moment, a significant reduction was found after day 6 for the delayed $(P=0.04)$ and after day 2 for the immediate loading group $(P=0.02)$. For the amount of swelling, this was, respectively, after day $4(P=0.003)$ and day 3 $(P=0.001)$. Over time, a significant reduction could be found for the amount of medication taken by the patient (Table 6): for the delayed loading group after day $6(P=0.04)$ and for the immediate loading group after day 3 $(P=0.001)$. Table 5 also shows the VAS score at the time of surgery and after 10 days. No difference for both groups was revealed over time.

No statistical differences could be shown between treatment groups on pain response (MPQ-DLV), treatment perception (VAS), number or kind of pain killers, or for the HRQOLI instrument. A post hoc power analysis was performed, and for most variables, there was clinically and statistically no difference $(\mathrm{N}$-factor $>6)$. However, there was a tendency for the delayed loading group to experience more postoperative discomfort (HRQOLI) for a longer period of time $(\mathrm{N}$-factor $<3$ ).

Table 5. Descriptive statistics of the HRQOL instrument and the VAS scores filled in at the time of surgery and at the evaluation meeting after 10 days

\begin{tabular}{|c|c|c|}
\hline Treatment group & Delayed & Immediate \\
\hline \multicolumn{3}{|l|}{ HRQOL Index } \\
\hline Day 1 & 27,$9 ; 29.5$ (SD 10.5) & $31.9 ; 30$ (SD 12.6) \\
\hline Day 2 & $27.1 ; 25$ (SD 11.8) & 25; 25 (SD 9.6) \\
\hline Day 3 & $27.4 ; 23$ (SD 13.5) & $25.4 ; 24$ (SD 10.4) \\
\hline Day 4 & $29.1 ; 24.5$ (SD 16.7) & $20.7 ; 21$ (SD 5.1) \\
\hline Day 5 & $23.6 ; 22$ (SD 8.2) & 19.3; 21 (SD 6.2) \\
\hline Day 6 & $22.1 ; 17$ (SD 9.1) & $19.1 ; 20$ (SD 4) \\
\hline Day 7 & $20 ; 17$ (SD 8.2) & 18.4; 19 (SD 4.9) \\
\hline \multicolumn{3}{|l|}{ VAS (In Office) } \\
\hline \multicolumn{3}{|l|}{ Mean pain $24 \mathrm{~h}$} \\
\hline After surgery & $1 ; 0$ (SD 1.4) & $5.9 ; 0$ (SD 12.1) \\
\hline 10 days & $1.8 ; 0$ (SD 2.7) & 7.4; 5 (SD 8.3) \\
\hline \multicolumn{3}{|c|}{ Pain during surgery } \\
\hline After surgery & 9.9; 6.5 (SD 11.2) & 18.7; 15 (SD 27) \\
\hline 10 days & $13.5 ; 4.5$ (SD 18.9) & 24.7; 9 (SD 30.9) \\
\hline \multicolumn{3}{|l|}{ Repeat procedure } \\
\hline After surgery & 9.6; 4 (SD 15.8) & $23.1 ; 18$ (SD 31.1) \\
\hline 10 days & $10 ; 5.5$ (SD 14.4) & $23.9 ; 15$ (SD 29.7) \\
\hline \multicolumn{3}{|c|}{ Duration procedure } \\
\hline After surgery & 9.0; 3.5 (SD 16.6) & 13.9; 6 (SD 21.2) \\
\hline 10 days & 15.2; 7 (SD 22.3) & $13.1 ; 10$ (SD 17) \\
\hline \multicolumn{3}{|c|}{ Recommend procedure } \\
\hline After surgery & $9.9 ; 4.5$ (SD 16) & $20.4 ; 6$ (SD 26.9) \\
\hline 10 days & $9.5 ; 4.5$ (SD 14) & 21.3; 5 (SD 30.2) \\
\hline
\end{tabular}

The HRQOL Index ranges from 0 to 75 , with higher scores on the HRQOLI being indicative of more postoperative discomfort and inconvenience in daily life. The mean, median, and standard deviation are presented. 


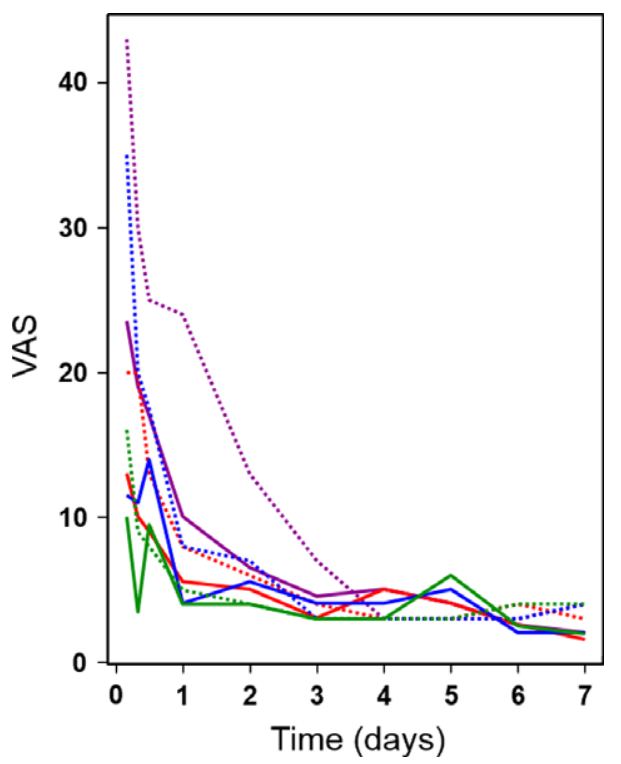

Fig. 4. Graphic showing the evolution over time of the VAS score. Green = amount of swelling, blue $=$ mean amount of pain at the moment, red = minimum amount of pain, and purple = maximum amount of pain are presented. Full line = delayed loading, interrupted line $=$ immediate loading.

\section{Discussion}

The accuracy data from the present study are comparable with other clinical studies on mucosa- or bone-supported stereolithographic guides in fully edentulous jaws (Pettersson et al. 2012; Arisan et al. 2013; D'Haese et al. 2012). If we compare the data with the results of a recent systematic review (Tahmaseb et al. 2014), taken into account the data from the in vivo studies, results are comparable as well (mean deviation at the entry $[1.12 \mathrm{~mm}$, Max.: $4.5 \mathrm{~mm}]$; at the apex $[1.39 \mathrm{~mm}$, Max.: $7.1 \mathrm{~mm}$ ] and mean angular deviation [3.89 , Max.: $21.16^{\circ}$ ]). Measurements in mesio-distal and bucco-lingual direction are also comparable to previous reports (Verhamme et al. 2013; Vercruyssen et al. 2014a).

The guided system tested in this study has some specific properties. The drill was guided with a sleeve, which was attached directly to the drill. A physic drill stop indicated the correct depth during the drilling procedure. For the implant placement, an implant holder
Table 6. Descriptive statistics of the pain medication

\begin{tabular}{|c|c|c|}
\hline Treatment group & Delayed & Immediate \\
\hline \multicolumn{3}{|l|}{ Pain medication } \\
\hline \multicolumn{3}{|l|}{ Day 1} \\
\hline No medication (\%) & 37.5 & 14.3 \\
\hline Paracetamol 500 mg 3/day (\%) & 25 & 28.6 \\
\hline >Paracetamol 500 mg 3/day (\%) & 12.5 & 28.6 \\
\hline Stronger pain medication (\%) & 25 & 28.6 \\
\hline \multicolumn{3}{|l|}{ Day 2} \\
\hline No medication (\%) & 50 & 57.1 \\
\hline Paracetamol 500 mg 3/day (\%) & 25 & 14.3 \\
\hline >Paracetamol 500 mg 3/day (\%) & 0 & 14.3 \\
\hline Stronger pain medication (\%) & 25 & 14.3 \\
\hline \multicolumn{3}{|l|}{ Day 3} \\
\hline No medication (\%) & 62.5 & 85.7 \\
\hline Paracetamol 500 mg 3/day (\%) & 12.5 & 14.3 \\
\hline >Paracetamol 500 mg 3/day (\%) & 0 & 0 \\
\hline Stronger pain medication (\%) & 25 & 0 \\
\hline \multicolumn{3}{|l|}{ Day 4} \\
\hline No medication (\%) & 75 & 85.7 \\
\hline Paracetamol 500 mg 3/day (\%) & 0 & 14.3 \\
\hline >Paracetamol 500 mg 3/day (\%) & 0 & 0 \\
\hline Stronger pain medication (\%) & 25 & 0 \\
\hline \multicolumn{3}{|l|}{ Day 5} \\
\hline No medication (\%) & 87.5 & 100 \\
\hline Paracetamol 500 mg 3/day (\%) & 0 & 0 \\
\hline$>$ Paracetamol 500 mg 3/day (\%) & 0 & 0 \\
\hline Stronger pain medication (\%) & 12.5 & 0 \\
\hline \multicolumn{3}{|l|}{ Day 6} \\
\hline No medication (\%) & 87.5 & 85.7 \\
\hline Paracetamol 500 mg 3/day (\%) & 12.5 & 0 \\
\hline$>$ Paracetamol 500 mg 3/day (\%) & 0 & 14.3 \\
\hline Stronger pain medication (\%) & 0 & 0 \\
\hline \multicolumn{3}{|l|}{ Day 7} \\
\hline No medication (\%) & 83.3 & 85.7 \\
\hline Paracetamol 500 mg 3/day (\%) & 16.7 & 0 \\
\hline >Paracetamol 500 mg 3/day (\%) & 0 & 14.3 \\
\hline Stronger pain medication (\%) & 0 & 0 \\
\hline
\end{tabular}

guided the implant in the correct position with a visual stop. In an in vitro study (Koop et al. 2012), we observed that the use of drillhold sleeve inserts gave for all measurements larger deviations than handhold sleeve inserts. In a recent randomized clinical trial /Vercruyssen et al. 2014b), we compared two other guided surgery systems. For both systems, handhold sleeve inserts were used. If we compare only the data for the mucosa-supported guides, comparable data could be found for the Materialise Universal ${ }^{\circledR}$ System (mean deviation at the entry $(1.2 \mathrm{~mm}$, range: $0.3-2.7 \mathrm{~mm})$ : at the apex of $1.6 \mathrm{~mm}$ (range: $0.5-3.0$ ), and angular deviation $\left(2.9^{\circ}\right.$ (range: $\left.\left.0.3-7.6^{\circ}\right)\right)$, the Facilitate $^{\mathrm{TM}}$ system (mean deviation at the entry $(1.4 \mathrm{~mm}$, range: $0.4-2.7 \mathrm{~mm})$ : at the apex of $1.6 \mathrm{~mm}$ (range: $0.2-3.3$ ), and angular deviation $\left(2.7^{\circ}\right.$ (range: $\left.0.2-6.4^{\circ}\right)$; and the Expertease $^{\mathrm{TM}}$ system (mean deviation at the entry (0.9 $\mathrm{mm}$, range: $0.1-4.45 \mathrm{~mm})$ : at the apex of $1.2 \mathrm{~mm}$ (range: $0.2-4.9 \mathrm{~mm}$ ), and angular deviation $\left(2.7^{\circ}\right.$ (range: $\left.0.0-6.6^{\circ}\right)$ ). So, although in vitro data showed larger deviations for the drill-hold sleeve, these differences seem to be clinically no longer relevant.

The improved accuracy obtained with guided implant surgery offers of course many advantages for the prosthodontist. Besides an improvement in the relationship between surgeon and prosthodontist, due to an improved planning and a prosthetic-driven approach, it also facilitates the immediate loading procedure. It even allows the use of a prefabricated restoration which can be delivered immediately after implant placement to improve patient satisfaction including comfort, function, and esthetics (De Bruyn et al. 2014; Pozzi et al. 2014). In this study, patients in the immediate loading group received their final prosthesis $24 \mathrm{~h}$ after implant placement. Because of preliminary ending of this study, the power to evaluate the patient-centered differences between treatment groups is limited. Furthermore, the postoperative discomfort for the patients in both treatment groups was low. Both groups were treated with a flapless protocol. And although it is generally accepted that flapless surgery gives less postoperative discomfort (Hultin et al. 2012), data from a recent randomized clinical trial (Vercruyssen et al. 2014c) revealed little difference in patient-centered outcome for mucosa- or bone-supported guided surgery.

Few studies report on the difference in patient-centered outcome between delayed and immediate loading. In a study of Nkenke et al. (2007), it is mentioned that the patients who received an immediate restoration wore this superstructure when the postoperative 
data were acquired and patients who received implants that were allowed to heal in a submerged fashion wore the surgical template at the pre- and postoperative data acquisitions. However, it is not discussed if this difference in prosthetic rehabilitation had an influence on the patient well-being. In this study, patients from the delayed group were not allowed to wear their prosthesis during the first week after surgery. This could be an explanation why there was a tendency for the delayed loading group to experience more postoperative discomfort (HRQOLI) for a longer period of time. For the other variables

\section{References}

Arisan, V., Karabuda, Z.C., Piskin, B. \& Ozdemir, T. (2013) Conventional Multi-Slice Computed Tomography (CT) and Cone-Beam CT (CBCT) for computer-aided implant placement. Part II: reliability of mucosa-supported stereolithographic guides. Clinical Implant Dental Related Research 15: 907-917.

De Bruyn, H., Raes, S., Ostman, P.O. \& Cosyn, J. (2014) Immediate loading in partially and completely edentulous jaws: a review of the literature with clinical guidelines. Periodontology 2000 66: 153-187.

D'Haese, J., Van De Velde, T., Elaut, L. \& De Bruyn, H. (2012) A prospective study on the accuracy of mucosally supported stereolithographic surgical guides in fully edentulous maxillae. Clinical Implant Dental Related Research 14: 293-303.

Hultin, M., Svensson, K.G. \& Trulsson, M. (2012) Clinical advantages of computer-guided implant placement: a systematic review. Clinical Oral Implants Research 23: 124-135.

Jacobs, R. \& Quirynen, M. (2014) Dental cone beam CT: justification for use in planning oral implant placement. Periodontology 2000 66: 203-213.

Koop, R., Vercruyssen, M., Vermeulen, K. \& Quirynen, M. (2012) Tolerance within the sleeve inserts of different surgical guides for guided implant surgery. Clinical Oral Implants Research 24: 630-634.

Lindeboom, J.A. \& van Wijk, A.J. (2010) A comparison of two implant techniques on patient-based outcome measures: a report of flapless vs. conventional flapped implant placement. Clinical Oral Implants Research 21: 366-370.

Maes, F., Collignon, A., Vandermeulen, D., Marchal, G. \& Suetens, P. (1997) Multimodality image registration by maximization of mutual information. IEEE Transactions on Medical Imaging 16: 187-198.

Melzack, R. (1975) The McGill Pain Questionnaire: major properties and scoring methods. Pain 1: 277-299.

Melzack, R. (2005) The McGill pain questionnaire: from description to measurement. Anesthesiology 103: 199-202.

Nkenke, E., Eitner, S., Radespiel-Troger, M., Vairaktaris, E., Neukam, F.W. \& Fenner, M. (2007) investigated in this study, no difference could be found between the delayed and the immediate loading group. So within the limitations of this study, there seems to be little advantage with an immediate loading protocol versus a delayed loading in the first days after surgery.

\section{Conclusion}

The accuracy of a novel CT-based guide (ExpertEase ${ }^{\mathrm{TM}}$ ) is comparable to accuracy data of other systems. Within the limitations of this study, no difference could be found in patient-centered outcome variables after immediate or delayed loading.

\section{Conflict of interest and source of funding statement}

There are no conflict of interests.

Oral implants were delivered free of charge by DENTSPLY Implants (Mölndal, Sweden). Stereolithographic guides were delivered free of charge by the Materialise Dental Company (Leuven, Belgium).
Patient-centred outcomes comparing transmucosal implant placement with an open approach in the maxilla: a prospective, non-randomized pilot study. Clinical Oral Implants Research 18: 197203.

Pettersson, A., Komiyama, A., Hultin, M., Nasstrom, K. \& Klinge, B. (2012) Accuracy of Virtually Planned and Template Guided Implant Surgery on Edentate Patients. Clinical Implant Dental Related Research 14: 527-537.

Pozzi, A., Tallarico, M., Marchetti, M., Scarfò, B. \& Esposito, M. (2014) Computer-guided versus freehand placement of immediately loaded dental implants: 1-year post-loading results of a multicentre randomised controlled trial. European Journal of Oral Implantology 7: 229-242.

Shugars, D.A., Benson, K., White, R.P., Jr, Simpson, K.N. \& Bader, J.D. (1996) Developing a measure of patient perceptions of short-term outcomes of third molar surgery. Journal of Oral Maxillofacial Surgery 54: 1402-1408.

Šidák, Z. (1967) Rectangular confidence regions for the means of multivariate normal distributions. Journal of American Statistical Association 62: 626-633.

Tahmaseb, A., Wismeijer, D., Coucke, W. \& Derksen, W. (2014) Computer technology applications in surgical implant dentistry: a systematic review. International Journal of Oral Maxillofacial Implants. 29: 25-42.

Van Assche, N., Vercruyssen, M., Coucke, W., Teughels, W., Jacobs, R. \& Quirynen, M. (2012) Accuracy of computer-aided implant placement. Clinical Oral Implants Research 23: 112-123.

Vercruyssen, M., Coucke, W., Naert, I., Jacobs, R. \& Quirynen, M. (2014a) Depth and lateral deviations in guided implant surgery: an RCT comparing guided surgery with mental navigation 1 or the use of a pilot-drill template. Clinical Oral Implants Research. doi: 10.1111/clr.12460. [Epub ahead of print].

Vercruyssen, M., Cox, C., Coucke, W., Naert, I., Jacobs, R. \& Quirynen, M. (2014b) An RCT comparing guided implant surgery (bone or mucosa supported) with mental navigation or the use of a pilot-drill template. Journal of Clinical Periodontology 41: 717-723.
Vercruyssen, M., De Laat, A., Coucke, W. \& Quirynen, M. (2014c) An RCT comparing patient-centred outcome variables of guided surgery (bone or mucosa supported) with conventional implant placement. Journal of Clinical Periodontology 41: 724-732.

Vercruyssen, M., Fortin, T., Widmann, G., Jacobs, R. \& Quirynen, M. (2014d) Different techniques of static/dynamic guided surgery: modalities and indications. Periodontology 2000 66: 214-227.

Vercruyssen, M., Hultin, M., van Assche, N., Svensson, K., Naert, I. \& Quirynen, M. (2014e) Static guided surgery: accuracy and efficacy. Periodontology 2000 66: 228-246.

Vercruyssen, M., Jacobs, R., van Assche, N. \& van Steenberghe, D. (2008) The use of CT scan based planning for oral rehabilitation by means of implants and its transfer to the surgical field: a critical review on accuracy. Journal of Oral Rehabilitation 35: 454-474.

Verhamme, L.M., Meijer, G.J., Boumans, T., De Haan, A.F., Bergé, S.J. \& Maal, T.J. (2013) A clinically relevant accuracy study of computer-planned implant placement in the edentulous maxilla using mucosa-supported surgical templates. Clinical Implant Dental Related Research 24: 1265-1272.

Verstreken, K., Van Cleynenbreugel, J., Marchal, G., Naert, I., Suetens, P. \& van Steenberghe D. (1996) Computer-assisted planning of oral implant surgery: a three-dimensional approach. International Journal of Oral Maxillofacial Implants 11: 806-810.

Verstreken, K., Van Cleynenbreugel, J., Martens, K., Marchal, G., van Steenberghe, G. \& Suetens P. (1998) An image-guided planning system for endosseous oral implants. IEEE Transactions on Medical Imaging 17: 842-852.

\section{Supporting Information}

Additional Supporting Information may be found in the online version of this article:

Appendix S1 CONSORT 2010 checklist of information to include when reporting a randomised trial 